\title{
El hilo de Ariadna: la importancia de estudiar la cárcel para las mujeres desde un enfoque de género
}

\author{
Graciela Sapriza \\ Universidad de la República. Facultad de Humanidades y Ciencias de la Educación. \\ Centro de Estudios Interdisciplinarios Uruguayos, Uruguay \\ gracielasapriza@gmail.com \\ Natalia Montealegre Alegría \\ Universidad de la República. Facultad de Humanidades y Ciencias de la Educación. \\ Centro de Estudios Interdisciplinarios Uruguayos / Área de Derechos Humanos - \\ Servicio Central de Extensión y Actividades en el medio (SCEAM), Uruguay \\ montealegre.alegria@gmail.com
}

Esta edición de Descentrada reúne un conjunto de artículos que aportan miradas disciplinarias e interdisciplinarias diversas sobre el fenómeno carcelario en la región del Río de la Plata y que comparten el interés en el género como categoría necesaria para el abordaje reflexivo y crítico respecto a lo punitivo.

La cárcel se concibió como una institución "resocializadora" y "rehabilitadora" para varones de sexo masculino. Pero el cúmulo de investigaciones sobre la temática demuestran que el género de las personas privadas de su libertad determina especificidades que, además de impactar en sus subjetividades y los ámbitos intra carcelarios, lo hacen también en las relaciones familiares y micro sociales que podrían clasificarse como “extra-muros". Esta constatación, de algún modo, implicó la construcción de un "tratamiento penitenciario científico" delimitando el campo de lo carcelario e incluyendo en el presente a diversas disciplinas, como el trabajo social, la medicina, la psiquiatría y la psicología, con un objetivo claramente terapéutico (Carranza, 2012). De esta manera, el sistema penitenciario se presentó tanto como una especie de "hospital que tendría la función de curar al delincuente mediante un tratamiento adecuado" (Daroqui, 2000, p. 117), como también una manera de eliminar al "enemigo interno". Es así que el paradigma positivista de la rehabilitación social ha estado claramente presente en el discurso penitenciario a lo largo del siglo pasado y parte del presente, mientras que su propuesta ha sido interpelada a la luz de las realidades concretas y su androcentrismo ha sido impugnado por las académicas feministas. Destacaremos que, en Uruguay, la reforma penitenciaria, iniciada en 2011, dio comienzo a un proceso de cambio de ese enfoque. Dentro de este escenario, se despliega el aporte de múltiples experiencias de investigación reunidas en ocasión de la convocatoria del presente dossier para constituir este hilo de Ariadna, centrado en dar cuenta, en una clave comparativa regional, de la importancia de estudiar la cárcel para las mujeres desde un enfoque de género.

El conjunto de artículos seleccionados aporta herramientas para decodificar parte de las relaciones de poder y la producción de subjetividades dentro y fuera del recinto carcelario, a través de diversos enfoques que abarcan desde el devenir histórico a la consideración de las condiciones de reclusión de las cárceles para mujeres actuales, atendiendo aspectos teórico metodológicos e interpelaciones éticas asociadas con la investigación dentro de este campo de problemas. 
$\mathrm{Al}$ abordar la historia de la cárcel resulta paradójico que no existiera, en algunos países, un lugar específico para prisionizar a las mujeres. Esa ausencia de un espacio donde alojar a las transgresoras plantea el interrogante sobre qué era considerado un "delito femenino". Se observa cómo los diferentes procedimientos (códigos, normas y protocolos) se dirigen casi exclusivamente al delincuente hombre/varón, en el tratamiento del delito y la pena, como si no existieran mujeres en conflicto con la ley penal. Incluso, en algunos países con un amplio proceso de secularización que alcanza extremos anticlericales, se encuentra que el cuidado y la vigilancia de las presas estuvo a cargo de la Orden del Buen Pastor de Angers, fundada en Francia por María Eufrasia Pelletier, cuya misión consistía en reformar a "la mujer de vida irregular". Su importancia para el continente resulta ineludible ya que se radicaron inicialmente en Chile y se extendieron por América del Sur desde fines del siglo XIX hasta el presente.

De acuerdo con lo anterior, el cuerpo del dossier toma como punto de partida textos que permiten articular diversos lenguajes, espacios y tiempos, desplegando un arco que se inicia en el período colonial y muestra cómo los dispositivos del castigo a las mujeres trasvasaban las barreras de clase y la ubicación geográfica.

El texto de Lía Quarlieri, “Castigos, fugas y resistencias femeninas en el Río de la Plata colonial. Mujeres indígenas y españolas en historias conectadas", aborda las implicancias del castigo corporal, para mostrar cómo mujeres indígenas y mujeres españolas se encontraron igualmente afectadas por políticas patriarcales disciplinares del cuerpo, más allá de diferencias de clase, etnia y lugar. La investigadora parte de dos casos judiciales suscitados a fines del siglo XVIII, en las reducciones guaraníes pos-jesuitas y en la ciudad de Buenos Aires, demostrando de qué modo los castigos físicos se vinculan con la conceptualización del cuerpo femenino, en ese contexto que tiene como sustento determinadas políticas de dominio colonial y tradiciones jurídicas.

Siguiendo esta línea histórica, a continuación, se incorporan dos artículos que, de diferentes formas, introducen a la institución del Buen Pastor. En primer lugar, el trabajo de Ornella Maritano y Melina Deangelis - "La cárcel correccional como agente cultural: una aproximación al Buen Pastor (Córdoba, siglos XIX-XX)" - retoma una investigación anterior y, en base al análisis de fuentes históricas, reconstruye las particularidades que asumió, en Córdoba, la Cárcel del Buen Pastor, que se diseñó específicamente para la población femenina durante fines del siglo XIX y comienzos del XX, contribuyendo a consolidar representaciones sobre la autoridad legítima en el ejercicio correccional de las mujeres, en torno a lo que las autoras caracterizan como "ideal de domesticidad".

En esta misma línea, Graciela Sapriza -en "Las ineludibles monjas del Buen Pastor en la cárcel de mujeres (Uruguay, 1898-1989)" - plantea una singular situación experimentada en el Uruguay, a lo largo del siglo XIX y XX, con la permanencia de la Orden del Buen Pastor al cuidado y control de las mujeres presas. Si bien se trata de una situación compartida con otros países sudamericanos donde la orden logró implantarse, fue más acuciante para Uruguay, país en pleno proceso de secularización hasta extremos anticlericales. Este dilema reaparece, en 1929, en el marco de un nuevo impulso reformista y se expresa a través de las disputas entre las damas católicas, obispos y religiosas frente a diputados, médicos y jóvenes abogadas. Sobre esos conflictos es que la autora exhibe las diferentes concepciones respecto a la criminalidad femenina y las estrategias pedagógicas trazadas por unos y otros. Según lo desarrollado, en el caso uruguayo, es el logro de un modelo femenino aceptado por la sociedad mesocrática lo que explicaría la permanencia de la Orden a través del siglo, aún durante el período de la dictadura cívico-militar. La “redención” a través de la moral o la re-adaptación al ideal de domesticidad que las monjas aplicaron a lo largo del siglo resulta compartido en sus aspectos políticos tanto en Argentina como en Uruguay, a través del control de los cuerpos como demuestran las autoras en estos primeros tres artículos.

Luego de este un primer bloque de contenidos, el dossier da paso al abordaje de situaciones vinculadas a la dinámica carcelaria de las mujeres en el presente. Sobre esas realidades, el trabajo de la antropóloga Cecilia Garibaldi Rivoir - "Derechos generizados. Encrucijadas morales y legales para pensar la maternidad en la cárcel” - toma como punto de partida su investigación etnográfica en la cárcel de madres con hijos/as de 
Montevideo y problematiza las tensiones entre distintos niveles de discusión y reflexión sobre el fenómeno para preguntarse cómo dialoga la normativa legal con las moralidades en torno al derecho de las madres a vivir con sus hijos/as en la cárcel, cómo se reflejan estas tensiones en las prácticas y discursos tanto de presas como de funcionarios/as de la cárcel, cuáles son los efectos de estas tensiones en las formas de ejercer la maternidad.

A continuación, habilitando una mirada comparativa de esta articulación entre dispositivo carcelario y maternidad, en "El derecho a maternar de las personas presas. Estrategias de vinculación de las personas madres detenidas con sus hijos/as fuera de la prisión”, Verónica Manquel, desde la psicología, contribuye a ampliar la mirada respecto a otras implicancias prácticas en la vida cotidiana de hijos e hijas de mujeres que son privadas de su libertad por conflictos con la ley. La autora se detiene en los sentidos y las relaciones sociales que intervienen en ese proceso y en los modos en que impactan en los vínculos madre/hijos-as. En este caso, el material analizado son entrevistas en profundidad realizadas por la autora a mujeres detenidas en el Complejo Penitenciario Federal IV (Ezeiza, Buenos Aires).

Finalmente, el último artículo, elaborado por antropólogas y una abogada, describe dos experiencias de taller: una con personal penitenciario y otra con mujeres recluidas en la Unidad Penitenciaria No 5 (Montevideo), problematizando los procesos de alterización presentes y el modo en que el dispositivo carcelario condiciona las posibilidades de una práctica feminista y el desarrollo de identidades múltiples. El aporte de Mesa Varela, Montealegre Alegría y Meza Tananta - "Abordajes interdisciplinarios de mujeres con mujeres: escenas de la vida carcelaria" - se sustenta en una experiencia con énfasis en los derechos humanos de las mujeres prisioneras, en un contexto en que el giro punitivo en el país y el crecimiento del movimiento feminista habilitan nuevas preguntas para el quehacer académico.

Pensar la prisión para las mujeres desde una perspectiva de género constituye un desafío que requiere trabajar en los huecos y silencios de los registros y, al mismo tiempo, supone intentar decodificar el "sentido de la pena" cuando esta se dirige a castigar en forma diferenciada a varones y mujeres. Visibilizar las particularidades de la privación de libertad en las subjetividades femeninas, la preeminencia de los estereotipos de género en el tratamiento carcelario, así como reconstruir su historia, implica un cambio de perspectiva de importancia mayor que necesariamente interpela el campo de estudios de lo punitivo en América Latina.

Abrimos este espacio con la intención de promover el intercambio, la reflexión y la producción académica sobre la prisión para mujeres, sabiendo que esa labor constituye un desafío. La diversidad de enfoques reunida en el número de Descentrada apuesta a un diálogo entre investigaciones que conlleve a ir delimitando un campo de estudio y, paralelamente, contribuya a la formación de nóveles investigadoras e investigadores en esta temática.

\section{ReFERENCiAS}

Carranza, E. (2012). Situación penitenciaria en América Latina y el Caribe ¿Qué hacer? Anuario de derechos humanos. Santiago de Chile: Universidad de Chile.

Daroqui, A. (2000). La cárcel en la universidad. En Fabre, A. y Nari, M. (comp.). Voces de mujeres encarceladas (pp.101-121). Buenos Aires: Catálogos. 